\title{
A New Approximation Related to the Error Function
}

\author{
By W. R. Schucany and H. L. Gray
}

In a recent note $\mathrm{R}$. G. Hart [1] gives the following expression:

(1)

$$
\begin{aligned}
P(x)= & \frac{\exp \left(-x^{2} / 2\right)}{x} \\
& \times\left[1-\frac{\left(1+b x^{2}\right)^{1 / 2} /\left(1+a x^{2}\right)}{P_{0} x+\left[P_{0}{ }^{2} x^{2}+\exp \left(-x^{2} / 2\right)\left(1+b x^{2}\right)^{1 / 2} /\left(1+a x^{2}\right)\right]^{1 / 2}}\right],
\end{aligned}
$$

where

$$
\begin{aligned}
P_{0} & =(\pi / 2)^{1 / 2} \\
a & =\frac{1+\left(1+6 \pi-2 \pi^{2}\right)^{1 / 2}}{2 \pi} \\
b & =2 \pi a^{2},
\end{aligned}
$$

which closely approximates

$$
F(x)=\int_{x}^{\infty} \exp \left(-t^{2} / 2\right) d t
$$

A new and more simple expression has been developed and may be employed for $x>2$. We shall define

$$
E(x)=\frac{x \exp \left(-x^{2} / 2\right)}{x^{2}+2}\left[\frac{x^{6}+6 x^{4}-14 x^{2}-28}{x^{6}+5 x^{4}-20 x^{2}-4}\right],
$$

and the motivation for such a definition may be found in [2] and [3].

It is readily apparent that $E(x)$ and $F(x)$ have the following properties in common:

1. For $x>2, E(x)$ is real, positive and finite.

2. For $x>2, d E / d x$ is real, negative and finite.

3. As $x \rightarrow \infty, E(x) \rightarrow 0$.

4. As $x \rightarrow \infty, x \exp \left(x^{2} / 2\right) E(x) \rightarrow 1$.

5. As $x \rightarrow \infty, d E / d x \rightarrow 0$.

The following table compares $E(x)$ to $P(x) . P(x)$ is a better fit than Hasting's approximation [4] for $x>2$ and $E(x)$ appears to be superior to $P(x)$. The relative error, $\epsilon_{\phi}(x)$ is the quantity selected as a basis for comparison and

$$
\epsilon_{\phi}(x)=\left|\frac{F(x)-\phi(x)}{F(x)}\right| \text {. }
$$

Received May 15, 1967. 
The numbers in parenthesis are powers of ten, e.g., $(-2) .33837=.33837 \times 10^{-2}$.

\begin{tabular}{rccc}
\hline$x$ & $F(x)$ & $\epsilon_{P}(x)$ & $\epsilon_{E}(x)$ \\
\hline 2 & $(-1) .57026$ & $(-3) .49$ & $(-2) .20$ \\
3 & $(-2) .33837$ & $(-3) .20$ & $(-5) .99$ \\
4 & $(-4) .79388$ & $(-4) .48$ & $(-4) .10$ \\
5 & $(-6) .71853$ & $(-5) .43$ & $(-5) .39$ \\
6 & $(-8) .24730$ & $(-5) .56$ & $(-5) .13$ \\
8 & $(-14) .15594$ & $(-5) .54$ & $(-7) .42$ \\
10 & $(-22) .19100$ & $(-5) .32$ & \\
\hline
\end{tabular}

For the range of values of $x$ given in the table the expression for $P$ is obviously more complicated than $E$ and, in fact, on a digital computer requires about thirty percent more computation time. Unfortunately, however, the worth of $E(x)$ is questionable for $x<2$, which is not the case for $P(x)$.

LTV Electrosystems, Inc.

Greenville, Texas

1. R. G. Hart, "A close approximation related to the error function," Math. Comp., v. 20, 1966, pp. 600-602. appear.)

2. H. L. GRAY, "A limiting case of the G-transformation," SIAM J. Numer. Anal. (To

3. H. L. Gray \& W. R. Schucany, "A new rational approximation to Mills' ratio," J. Amer. Statist. A ssoc. (To appear.)

4. C. Hastings, Approximations for Digital Computers, Princeton Univ. Press, Princeton, N. J., 1955, p. 167. MR 16, 963.

\title{
Improvement in Recurrence Techniques for the Computation of Bessel Functions of Integral Order
}

\author{
By Fr. Mechel
}

The Bessel functions satisfy recurrence relations which are very convenient for the generation of these functions, especially when a great number of functions with varying index is needed, [1], [2], [3].

To start with the spherical Bessel functions $j_{n}(x), y_{n}(x)$, the recurrence relation

$$
f_{n-1}(x)+f_{n+1}(x)=((2 n+1) / x) f_{n}(x)
$$

is valid, which can be used either in the upward direction of the index $n$ or in the downward direction. For the generation of the spherical Neumann functions $y_{n}(x)$ it must be used in the upward direction with the starting functions

$$
y_{0}(x)=-\cos x / x \text { and } y_{1}(x)=-\sin x / x-\cos x / x^{2} .
$$

The computation of the spherical Bessel function $j_{n}(x)$ with Eq. (1) is more difficult, since now the recurrence relation must be used in the downward direction.

Received February 17, 1967. 